\title{
AN ALGORITHM FOR MULTIPARAMETRIC 0-1-INTEGER PROGRAMMING PROBLEMS RELATIVE TO A GENERALIZED MIN MAX OBJECTIVE FUNCTION
}

\author{
José Luis Quintero $^{1}$ And Alejandro Crema ${ }^{2}$
}

\begin{abstract}
The multiparametric 0-1-Integer Programming (0-1-IP) problem relative to the objective function is a family of $0-1$-IP problems which are related by having identical constraint matrix and righthand-side vector. In this paper we present an algorithm to perform a complete multiparametric analysis relative to a generalized min max objective function such that the min sum and min max are particular cases.
\end{abstract}

Keywords. 0-1-Integer Programming, multiparametric programming, Bottleneck problem.

Mathematics Subject Classification. 90C10, 90C31.

\section{INTRODUCTION}

Uncertainty in the data appears in most real 0-1-Integer Programming (0-1-IP) problems. Researchers have proposed several ideas in the literature in order to solve problems under uncertainty: stochastic programming and robust optimization for example. An alternative approach is to use parametric programming, which address the presence of an uncertain parameter, and multiparametric programming which considers the presence of two or more uncertain parameters. Greenberg [11] published an annotated bibliography for post-solution analysis including parametric IP problems. Greenberg's bibliography is available on the

Received October 17, 2007. Accepted July 3, 2008.

1 Departamento de Matemáticas Aplicadas, Facultad de Ingeniería, Universidad Central de Venezuela, Apartado 47002, Caracas 1041-A, Venezuela; quinteroj1@tutopia.com

2 Escuela de Computación, Facultad de Ciencias, Universidad Central de Venezuela, Apartado 47002, Caracas 1041-A, Venezuela; acrema@kuaimare.ciens.ucv.ve 
World Wide Web (WWW) [12]. Another bibliography available on the WWW, that includes references on parametric and multiparametric IP problems, is due to Arsham [4]. A detailed literature review in parametric IP problems can be found in Jenkins [17] and recently in Li and Ierapetritou [19] including multiparametric IP problems.

Jenkins [14-16] has presented a very simple approach to solve parametric IP problems based on Geoffrion and Nauss [10]. His methods work by solving an appropriate sequence of non-parametric problems and joining the solutions to complete the parametrical analysis.

We have used the Jenkins's approach in order to design algorithms to solve multiparametric 0-1-IP problems relative to the right-hand-side vector (Crema [6]), the min sum objective function (Crema [7]) and the constraint matrix (Crema [8]). The perturbation of the right-hand-side vector, the min sum objective function and the constraint matrix was considered by Crema [9] for 0-1-IP problems and by Li and Ierapetritou [19] for Mixed Integer Programming (MIP) problems.

A theoretical and algorithmic study for parametric 0-1-IP problems relative to the min sum objective function, including complexity results, have been written by Thiongane et al. [28]. The multiparametric problem relative to the min max objective function have been recently considered (Quintero and Crema [23]).

In this paper we present an approach, that can be viewed as a generalization of our previous work (Quintero and Crema [23]), to solve multiparametric 0-1-IP problems relative to a generalized min max objective function that includes the min sum and min max as particular cases.

Let $L \in \mathbb{R}^{p}, U \in \mathbb{R}^{p}$ with $L \leq U$, let $D \in \mathbb{R}^{q \times p}, d \in \mathbb{R}^{q}$ and let $\Omega=\left\{\theta \in \mathbb{R}^{p}\right.$ : $L \leq \theta \leq U, D \theta \leq d\}$, let $b \in \mathbb{R}^{m}, A \in \mathbb{R}^{m \times n}$ and let $X=\{x: A x \leq b, \quad x \in$ $\left.\{0,1\}^{n}\right\}$.

The multiparametric 0-1-IP problem relative to the objective function is a family of 0-1-IP problems which are related by having identical constraint matrix and right-hand-side vector. A member of the family is defined as

$$
(P(\theta)) \quad \min \phi(\theta, x) \quad \text { s.t. } \quad x \in X
$$

where $\phi$ is a continuous function on $\Omega$ for all $x \in X, \theta$ is the vector of parameters, $\theta \in \Omega \subseteq \mathbb{R}^{p}$ and $X$ is the set of feasible solutions which does not depend on the vector $\theta$. Let us suppose that $\Omega \neq \emptyset$ and $X \neq \emptyset$.

The objective function considered in this paper is defined as follows:

$$
\phi(\theta, x)=\max \left\{\left(F^{(1)} \theta\right)^{t} x+d^{(1)^{t}} \theta+c^{(1)^{t}} x, \cdots,\left(F^{(k)} \theta\right)^{t} x+d^{(k)^{t}} \theta+c^{(k)^{t}} x\right\}
$$

where:

$F^{(j)} \in \mathbb{R}^{n \times p}, d^{(j)} \in \mathbb{R}^{p}, c^{(j)} \in \mathbb{R}^{n} \quad(j=1, \cdots, k)$ and $k$ is the number of functions used to define $\phi$ that depends on the particular case considered. 
The min sum and min max are particular cases as follows. Let $k=1, p=$ $n, d^{(1)}=0, c^{(1)}=0, F^{(1)}=I$, then $\phi(\theta, x)=\theta^{t} x$ and we have the min sum case. Let $k=n, p=n,\left(F^{(j)} \theta\right)^{t}=\left(0, \cdots, \theta_{j}, \cdots, 0\right), d^{(j)}=0, c^{(j)}=0,(j=1, \cdots, k)$, then $\phi(\theta, x)=\max \left\{\theta_{1} x_{1}, \cdots, \theta_{n} x_{n}\right\}$ and we have the min max case. We consider other interesting cases in our computational experience.

Note that since $X$ is a finite set then there exists an optimal solution for $P(\theta)$ for all $\theta \in \Omega$. All the problems considered in this paper have optimal solutions.We use the following standard notation: if $T$ is an optimization problem $v(T)$ denotes its optimal value.

We say that $x^{(1)}, \cdots, x^{(r)}$ is a multiparametrical solution if:

(i) $x^{(i)} \in X$ for all $i=1, \cdots, r$;

(ii) $v(P(\theta))=\min \left\{\phi\left(\theta, x^{(1)}\right), \cdots, \phi\left(\theta, x^{(r)}\right)\right\}$ for all $\theta \in \Omega$.

Note that the index $r$ denotes the number of solutions used to define the multiparametrical solution.

In Section 2 we present the theory that allows us to design the algorithm presented in the same section. In Section 3 we present a general approach to write as a 0-1-MIP problem a non-linear 0-1-MIP problem that appears if we use our algorithm. Computational experience is presented in Section 4. A summary and further extensions is given in Section 5 .

\section{Theoretical RESUlts AND THE ALGORITHM}

This section is a summary of our previous results in solving multiparametric 0-1-IP problems relative to the objective function (Quintero and Crema [23]) that are independent of the particular case of $\phi$ to be considered.

Suppose that $x^{(i)} \in X$ for all $i=1, \cdots, r$ and let $g^{(r)}(\theta)$ be an upper bound function of $v(P(\theta))$ defined by $g^{(r)}(\theta)=\min \left\{\phi\left(\theta, x^{(1)}\right), \cdots, \phi\left(\theta, x^{(r)}\right)\right\}$. Note that for all $\theta \in \Omega$ we have $g^{(r)}(\theta) \geq v(P(\theta))$ and if $x^{(r+1)} \in X$ then $g^{(r+1)}(\theta) \leq g^{(r)}(\theta)$.

Let $Q^{(r)}$ be a problem in $(\theta, x, y, z)$ defined as:

$$
\begin{gathered}
\left(Q^{(r)}\right) \max \quad y-z \text { s.t. } \theta \in \Omega, x \in X \\
y=g^{(r)}(\theta) \\
z=\phi(\theta, x) \\
y \in \mathbb{R}, z \in \mathbb{R} .
\end{gathered}
$$

Observe that $\theta$ is a vector of decision variables in $Q^{(r)}$.

Note that with $Q^{(r)}$ we are looking for the maximal difference between $v(P(\theta))$ and $g^{(r)}(\theta)$. If the maximal difference is zero then we have found $v(P(\theta))$ for all $\theta \in \Omega$ and the analysis was completed, otherwise our algorithm finds $x^{(r+1)}$ and $\theta^{*}$ such that: 
(i) $g^{(r+1)}(\theta) \leq g^{(r)}(\theta)$ for all $\theta \in \Omega$

(ii) $g^{(r+1)}\left(\theta^{*}\right)<g^{(r)}\left(\theta^{*}\right)$.

Note that this approach has been presented in Crema [7] and Quintero and Crema [23] in a different context.

Lemma 2.1. (i) There exists an optimal solution for $Q^{(r)}$. (ii) $v\left(Q^{(r)}\right) \geq 0$. (iii) If $v\left(Q^{(r)}\right)=0$ then $v(P(\theta))=g^{(r)}(\theta)$ for all $\theta \in \Omega$. (iv) If $\left(\theta^{*}, x^{(r+1)}\right)$ is an optimal solution for $Q^{(r)}$ then $x^{(r+1)}$ is an optimal solution for $P\left(\theta^{*}\right)$. (v) Let $\left(\theta^{*}, x^{(r+1)}\right)$ be an optimal solution for $Q^{(r)}$. If $v\left(Q^{(r)}\right)>0$ then $x^{(i)}$ is not an optimal solution for $P\left(\theta^{*}\right)$ for all $i=1, \cdots, r$ and $g^{(r+1)}\left(\theta^{*}\right)<g^{(r)}\left(\theta^{*}\right)$.

The proof of Lemma 2.1 may be seen in Quintero and Crema [23]. Since $X$ is a finite set, the lemma proves that the next algorithm (the multiparametric algorithm) provide us a complete multiparametrical analysis:

Step 0. Find $\theta^{(1)} \in \Omega$. Solve $P\left(\theta^{(1)}\right)$. Let $x^{(1)}$ be an optimal solution.

Step 1. $r=1$

Step 2. Solve $Q^{(r)}$ and let $\left(\theta^{*}, x^{(r+1)}\right)$ be an optimal solution.

Step 3. If $v\left(Q^{(r)}\right)=0$ STOP (with $v(P(\theta))=g^{(r)}(\theta)$ for all $\theta \in \Omega$ ), otherwise let $r=r+1$ and return to step 2 .

In the min sum case $P\left(\theta^{(1)}\right)$ is a 0 -1-IP problem. With $\phi$ the objective function considered in this paper $P\left(\theta^{(1)}\right)$ may be rewritten as a 0 -1-MIP problem. In order to use the algorithm based on Lemma 2.1 we need an algorithm to solve $Q^{(r)}$ that was presented as a non-linear 0-1-MIP problem.

\section{An approach to solve $Q^{(r)}$}

The main contribution of this paper is an approach to solve $Q^{(r)}$ in the general case presented.

Problem $Q^{(r)}$ to be solved was defined as follows:

$$
\begin{aligned}
\left(Q^{(r)}\right) \max & y-z \text { s.t. } \theta \in \Omega, x \in X \\
& y=g^{(r)}(\theta) \\
& z=\phi(\theta, x) \\
y & \in \mathbb{R}, z \in \mathbb{R} .
\end{aligned}
$$

We present a sequence of problems that are equivalent to $Q^{(r)}$ until we reach an appropriate model to be solved as a 0-1-MIP problem.

We use the definition of the upper bound function, $g^{(r)}(\theta)$, and the definition of $\phi(\theta, x)$ in order to rewrite $Q^{(r)}$ as follows:

$$
\left(Q 1^{(r)}\right) \quad \max y-z \text { s.t. } \theta \in \Omega, \quad x \in X
$$




$$
\begin{gathered}
y=\min \left\{\max \left\{\left(F^{(j)} \theta\right)^{t} x^{(i)}+d^{(j)^{t}} \theta+c^{(j)^{t}} x^{(i)}: j=1, \cdots, k\right\}: i=1, \cdots, r\right\} \\
z=\max \left\{\left(F^{(j)} \theta\right)^{t} x+d^{(j)^{t}} \theta+c^{(j)^{t}} x: j=1, \cdots, k\right\} \\
y \in \mathbb{R}, z \in \mathbb{R} .
\end{gathered}
$$

Let $\left(Q 2^{(r)}\right)$ be a problem in $(\theta, x, y, z)$ defined as follows:

$$
\begin{gathered}
\left(Q 2^{(r)}\right) \quad \max y-z \text { s.t. } \theta \in \Omega, \quad x \in X \\
y \leq\left(F^{(j)} \theta\right)^{t} x^{(i)}+d^{(j)^{t}} \theta+c^{(j)^{t}} x^{(i)} \text { for some } j \in\{1, \cdots, k\} \quad(i=1, \cdots, r) \\
z \geq\left(F^{(j)} \theta\right)^{t} x+d^{(j)^{t}} \theta+c^{(j)^{t}} x \quad(j=1, \cdots, k) \\
y \in \mathbb{R}, z \in \mathbb{R} .
\end{gathered}
$$

Since maximization is the optimization criterion then problems $Q 1^{(r)}$ and $Q 2^{(r)}$ are equivalent: $(\theta, x, y, z)$ is an optimal solution to $Q 1^{(r)}$ if and only if $(\theta, x, y, z)$ is an optimal solution to $Q 2^{(r)}$ and $v\left(Q 1^{(r)}\right)=v\left(Q 2^{(r)}\right)$.

Finally we use 0-1 auxiliary variables in order to rewrite the constraints relative to $y$. Let $Q 3^{(r)}$ be a problem in $(\theta, x, y, z, w)$ defined as follows:

$$
\begin{gathered}
\left(Q 3^{(r)}\right) \quad \max \quad y-z \text { s.t. } \theta \in \Omega, x \in X \\
y \leq \sum_{j=1}^{k}\left(\left(F^{(j)} \theta\right)^{t} x^{(i)}+d^{(j)^{t}} \theta+c^{(j)^{t}} x^{(i)}\right) w_{j}^{(i)} \quad(i=1, \cdots, r) \\
\sum_{j=1}^{k} w_{j}^{(i)}=1 \quad(i=1, \cdots, r) \\
w_{j}^{(i)} \in\{0,1\} \quad(i=1, \cdots, r) \quad(j=1, \cdots, k) \\
z \geq\left(F^{(j)} \theta\right)^{t} x+d^{(j)^{t}} \theta+c^{(j)^{t}} x \quad(j=1, \cdots, k) \\
y \in \mathbb{R}, z \in \mathbb{R} .
\end{gathered}
$$

If $(\theta, x, y, z, w)$ is an optimal solution to $Q 3^{(r)}$ then $(\theta, x, y, z)$ is an optimal solution to $Q 2^{(r)}$. If $(\theta, x, y, z)$ is an optimal solution to $Q 2^{(r)}$ then $(\theta, x, y, z, w)$ is an optimal solution to $Q 3^{(r)}$ with $w$ defined as follows:

(i) let $s$ be an index such that:

$$
\begin{aligned}
& \max \left\{\left(F^{(j)} \theta\right)^{t} x^{(i)}+d^{(j)^{t}} \theta+c^{(j)^{t}} x^{(i)}: j=1, \cdots, k\right\}=\left(F^{(s)} \theta\right)^{t} x^{(i)} \\
+ & \left.d^{(s)^{t}} \theta+c^{(s)^{t}} x^{(i)}\right) \text {, with the ties broken arbitrarily, }
\end{aligned}
$$

(ii) $w_{s}^{(i)}=1$ and $w_{j}^{(i)}=0$ if $j \neq s(i=1, \cdots, r$ and $j=1, \cdots, k)$.

Hence, $Q 2^{(r)}$ and $Q 3^{(r)}$ are equivalent problems and $v\left(Q 2^{(r)}\right)=v\left(Q 3^{(r)}\right)$. Therefore $Q^{(r)}$ and $Q 3^{(r)}$ are equivalent problems and $v\left(Q^{(r)}\right)=v\left(Q 3^{(r)}\right)$

Note that $Q 3^{(r)}$ includes non-linear terms. However, all of them may be rewritten with an standard method (Adams, Forrester and Glover [2]) in order to solve $Q 3^{(r)}$ as a $0-1-$ MIP problem: 
For all $i=1, \cdots, r$ and $j=1, \cdots, k$ use $\alpha_{j}^{(i)} w_{j}^{(i)}$ instead of $\left(F^{(j)} \theta\right)^{t} x^{(i)} w_{j}^{(i)}$ where $\alpha_{j}^{(i)}$ is a new continuous variable and add to $Q 3^{(r)}$ the constraints:

$$
\begin{gathered}
V_{j}^{(i)}\left(1-w_{j}^{(i)}\right) \leq\left(F^{(j)} \theta\right)^{t} x^{(i)}-\alpha_{j}^{(i)} \leq S_{j}^{(i)}\left(1-w_{j}^{(i)}\right) \\
V_{j}^{(i)} w_{j}^{(i)} \leq \alpha_{j}^{(i)} \leq S_{j}^{(i)} w_{j}^{(i)} \\
\alpha_{j}^{(i)} \in \mathbb{R}(i=1, \cdots, r)(j=1, \cdots, k)
\end{gathered}
$$

where $V_{j}^{(i)}$ and $S_{j}^{(i)}$ are bounds such that:

$$
\begin{aligned}
& S_{j}^{(i)} \geq \max \left\{\left(F^{(j)} \theta\right)^{t} x^{(i)}: \theta \in \Omega\right\} \\
& V_{j}^{(i)} \leq \min \left\{\left(F^{(j)} \theta\right)^{t} x^{(i)}: \theta \in \Omega\right\} .
\end{aligned}
$$

Terms $d^{(j)^{t}} \theta w_{j}^{(i)}$ and $\left(F^{(j)} \theta\right)^{t}{ }_{l} x_{l}$ may be replaced by using the same method for all $i=1, \cdots, r, j=1, \cdots, k$ and $l=1, \cdots, n$.

We present the standard method because it is appropriate to the general objective function considered, however, others methods, that depend on the particular case of objective function, may be used to replace the non-linear terms (Adams et al. [2], Adams and Forrester [1], Oral and Kettani [22]).

To the best of our knowledge there are no other algorithms to be applied in the general case considered. Our algorithm may be implemented by using any software capable of solving MIP problems.

\section{Computational eXperience}

Previous computational experiences in the min sum case and min max cases were presented in Crema [7] and Quintero and Crema [23]. The problems considered were the multiconstrained knapsack problem, with the min sum objective, and the bottleneck generalized assignment problem, with the min max objective function (Martello and Toth [20]). In both cases the linearization process of $Q^{(r)}$ did not use the standard method presented in this paper because of the particular objective function considered.

Our algorithm has been implemented in $\mathrm{C}++$ by using the OSL package of IBM $[13,21]$ that uses a Branch and bound algorithm based on linear relaxations to solve MIP problems. The new experiments with the general objective function were performed on a PC Pentium IV with $2 \mathrm{Ghz}$ and $256 \mathrm{MB}$ of RAM.

The problem considered in this paper is the $m$-Bottleneck assignment $(m$-BA) problem as follows:

Given $n$ tasks and $m$ agents, the penality, $c_{i j}$, corresponding to the assignment of the task $j$ to agent $i$ and given $a_{i j} \in\{0,1\}$ such that the task $j$ may be assigned to agent $i$ if and only if $a_{i j}=1(i=1, \cdots, m$ and $j=1, \cdots, n)$, the $m$-BA problem 
is to assign each task to one agent so that the maximum penality of the agents is minimized where the penality of the agent $i$ is computed as $\sum_{j \text { assigned to } i} \theta_{i j}$.

By introducing binary variables $x_{i j}$ with $x_{i j}=1$ if and only if task $j$ is assigned to agent $i$, the problem can be formulated as follows:

$$
\begin{aligned}
& \min \max \left\{\sum_{j=1}^{n} c_{i j} x_{i j}: i=1, \cdots, m\right\} \text { s.t. } \\
& \sum_{i=1}^{m} a_{i j} x_{i j}=1(j=1, \cdots, n) \\
& x_{i j} \in\{0,1\}, \quad(i=1, \cdots m)(j=1, \cdots, n) .
\end{aligned}
$$

Let us suppose that some of the penalities are uncertain parameters and then we rewrite the problem by using $\theta_{i j}$ instead of $c_{i j}$. Let $K$ be the index set of elements of the objective function that are uncertain parameters.

The experiments were designed with $\Omega=\left\{\theta_{i j}:(1-\beta) c_{i j} \leq \theta_{i j} \leq(1+\right.$ $\beta) c_{i j}$ for all $(i, j) \in K, \quad \theta_{i j}=c_{i j}$ for all $\left.(i, j) \notin K\right\}$ where $0<\beta<1$ is a parameter used to define the level of perturbation.

The indexes that belong to $K$ were randomly selected from the set $\{(i, j)$ : $a_{i j}=1, i=1, \cdots, n$ and $\left.j=1, \cdots, m\right\}$.

In our experimets the elements $c_{i j}$ and $a_{i j}$ were generated as follows:

(i) Uncorrelated case: the elements $c_{i j}$ were drawn from a uniform distribution on $[1, c \max ]$ with $c \max >1$. If $c_{j_{1} j} \leq c_{j_{2} j} \leq \cdots \leq c_{j_{m} j}$ then we use $a_{j_{1} j}=a_{j_{2} j}=1$ and $a_{i j}=0$ if $i \notin\left\{j_{1}, j_{2}\right\}$.

(ii) Correlated case: given $n$ towns (the tasks) and $m$ service centers (the agents), the distance, $d_{i j}$, from town $j$ until service center $i$ and the population, $P_{j}$, of town $j$, then the penality, $c_{i j}$, corresponding to the assignment of the town $j$ to service center $i$ is defined as $c_{i j}=d_{i j} P_{j}$. The location of town $j$ and service center $i$ were drawn from a uniform distribution on the square $[0,1] \times[0,1]$ and $d_{i j}$ is the euclidean distance from the location of $i$ until the location of $j(i=1, \cdots, m$ and $j=1, \cdots, n)$. The elements $P_{j}$ were drawn from a uniform distribution on $[1, P \max ]$ with $P \max >1$. If $d_{j_{1} j} \leq d_{j_{2} j} \leq \cdots \leq d_{j_{m} j}$ then we use $a_{j_{1} j}=a_{j_{2} j}=1$ and $a_{i j}=0$ if $i \notin\left\{j_{1}, j_{2}\right\}$.

The results are reported in Table 1 (uncorrelated case) and Table 2 (correlated case). The notation used in the tables is as follows: $p$ is an index to identify the problem, $r$ is the number of $Q^{(r)}$ problems solved in order to complete the multiparametrical analysis, $S i 1$ is the number of simplex iterations computed to 
TABLE 1. Computational experience to the uncorrelated case.

\begin{tabular}{|c|c|c|c|c|c|c|c|c|c|c|c|}
\hline$p$ & $m$ & $n$ & $n_{K}$ & $c \max$ & $\beta$ & $r$ & Si1 & N1 & Si & N & $t$ \\
\hline 1 & 2 & 10 & 10 & 1000 & 0.20 & 2 & 18 & 7 & 1507 & 99 & 13.130 \\
2 & & 20 & & & & 3 & 32 & 10 & 4317 & 332 & 356.149 \\
3 & & 30 & & & & 4 & 58 & 19 & 926836 & 17577 & 9145.388 \\
\hline 4 & 2 & 10 & 10 & 1000 & 0.05 & 2 & 23 & 9 & 456 & 22 & 4.032 \\
5 & & & & & 0.10 & 2 & 22 & 10 & 617 & 25 & 12.167 \\
6 & & & & & 0.15 & 4 & 25 & 11 & 832 & 31 & 13.672 \\
7 & & & & & 0.25 & 4 & 27 & 15 & 706 & 96 & 15.523 \\
8 & & & & & 0.30 & 5 & 21 & 9 & 3618 & 362 & 315.517 \\
9 & & & & & 0.35 & 5 & 20 & 6 & 1622 & 92 & 25.512 \\
10 & & & & & 0.40 & 8 & 18 & 5 & 3526 & 331 & 427.735 \\
\hline 11 & 2 & 10 & 5 & 1000 & 0.20 & 2 & 19 & 5 & 435 & 20 & 9.541 \\
12 & & & 15 & & & 3 & 24 & 9 & 945 & 45 & 24.478 \\
13 & & & 20 & & & 4 & 24 & 10 & 1566 & 91 & 36.376 \\
\hline 14 & 2 & 10 & 10 & 10 & 0.20 & 3 & 20 & 6 & 736 & 36 & 8.151 \\
15 & & & & 100 & & 3 & 19 & 3 & 625 & 28 & 6.292 \\
16 & & & & 10000 & & 4 & 19 & 2 & 1240 & 46 & 15.261 \\
\hline 17 & 3 & 20 & 10 & 1000 & 0.20 & 7 & 35 & 12 & 934622 & 26333 & 9532.336 \\
18 & 5 & & & & & 10 & 69 & 25 & 1822633 & 61226 & 17457.672 \\
\hline
\end{tabular}

solve $P\left(\theta^{1}\right)$, N1 is the number of nodes generated by the branch and bound algorithm to solve $P\left(\theta^{1}\right), S i$ is the number of simplex iterations computed to solve $P\left(\theta^{1}\right), Q^{(1)}, \cdots Q^{(r)}$. The number of nodes generated by the branch and bound algorithm to solve $P\left(\theta^{1}\right), Q^{(1)}, \cdots Q^{(r)}$ is denoted with $N$ and $t$ is the CPU time in seconds to solve $P\left(\theta^{1}\right), Q^{(1)}, \cdots Q^{(r)}$. Both, $S i 1$ and $S i$, include the number of simplex iterations computed to solve the relaxations of subproblems in the branch and bound algorithm.

Non-linear terms were replaced following a generalization of the procedure used to the min max case (Quintero and Crema [23]) based on Oral and Kettanni [22], instead of the standard method, in order to generate a model with fewer constraints.

The values of $\theta_{i j}^{(1)}$ were defined as follows:

$\theta_{i j}^{(1)}=c_{i j}$ if $(i, j) \notin K$ and $\theta_{i j}^{(1)}=(1-\beta) c_{i j}$ if $(i, j) \in K(i=1, \cdots, n)$, $(j=1, \cdots, m)$.

Let $n_{K}$ be the cardinality of $K$. The experiments were designed in order to evaluate the perfomance of the algorithm as $m$ increases (problems $17-18$ and 37-39), $n$ increases (problems 1-3 and 19-23), $n_{K}$ increases (problems 11-13 and 31-33), $c$ max increases (problems 14-16), P max increases (problems 34-36), or $\beta$ increases (problems $4-10$ and 24-30). As we can expect the effort increases as the dimensions $\left(m\right.$ and $n$ ), the number of parameters $\left(n_{K}\right)$ and the level of perturbation $(\beta)$ increase. As we hope the uncorrelated problems seem to be more 
TABLE 2. Computational experience to the correlated case.

\begin{tabular}{|c|c|c|c|c|c|c|c|c|c|c|c|}
\hline$p$ & $m$ & $n$ & $n_{K}$ & $P \max$ & $\beta$ & $r$ & $\mathrm{Si}$ & $\mathrm{N} 1$ & $\mathrm{Si}$ & $\mathrm{N}$ & $t$ \\
\hline 19 & 3 & 10 & 10 & 1000 & 0.20 & 5 & 51 & 21 & 6564 & 336 & 3.241 \\
20 & & 20 & & & & 8 & 98 & 39 & 7031 & 521 & 6.127 \\
21 & & 30 & & & & 10 & 265 & 76 & 13259 & 822 & 17.230 \\
22 & & 40 & & & & 15 & 411 & 95 & 21346 & 1336 & 234.216 \\
23 & & 50 & & & & 17 & 623 & 121 & 45165 & 3845 & 2561.377 \\
\hline 24 & 3 & 10 & 10 & 1000 & 0.05 & 5 & 67 & 22 & 7126 & 429 & 5.040 \\
25 & & & & & 0.10 & 7 & 78 & 29 & 8655 & 784 & 9.954 \\
26 & & & & & 0.15 & 7 & 92 & 26 & 11278 & 832 & 51.265 \\
27 & & & & & 0.25 & 6 & 54 & 17 & 9188 & 812 & 34.167 \\
28 & & & & & 0.30 & 11 & 99 & 27 & 19424 & 1239 & 191.637 \\
29 & & & & & 0.35 & 10 & 156 & 34 & 17823 & 1038 & 125.527 \\
30 & & & & & 0.40 & 14 & 178 & 36 & 21296 & 1495 & 436.163 \\
\hline 31 & 3 & 10 & 5 & 1000 & 0.20 & 5 & 55 & 19 & 7527 & 362 & 7.362 \\
32 & & & 15 & & & 8 & 94 & 35 & 13349 & 923 & 74.396 \\
33 & & & 20 & & & 15 & 114 & 47 & 25562 & 2156 & 924.169 \\
\hline 34 & 3 & 10 & 10 & 10 & 0.20 & 7 & 45 & 19 & 13636 & 938 & 7.321 \\
35 & & & & 100 & & 8 & 56 & 17 & 16723 & 1326 & 54.252 \\
36 & & & & 10000 & & 10 & 72 & 25 & 20362 & 1934 & 152.241 \\
\hline 37 & 5 & 30 & 10 & 1000 & 0.20 & 16 & 158 & 37 & 56578 & 4020 & 932.241 \\
38 & 7 & & & & & 21 & 466 & 64 & 96377 & 7625 & 9448.478 \\
39 & 9 & & & & & 26 & 593 & 91 & 674744 & 59272 & 23363.644 \\
\hline
\end{tabular}

difficult than the correlated problems. However, more problems should be solved to make definitive conclusions.

\section{Summary AND FURTHER EXTENSIONS}

We designed and implemented an algorithm to solve the multiparametric 0-1-IP problem relative to a very general objective function (min sum and min max are particular cases). Computational experience was presented for $m$-BA problems with uncorrelated and correlated data. Our algorithm works by choosing an appropriate finite sequence of non-parametric MIP problems in order to obtain a complete multiparametrical analysis and this explains that the computer storage was not a problem for our algorithm, that is: if we can solve the non-parametric $P\left(\theta^{(1)}\right)$ problem then we can expect no problems to perform a complete multiparametrical analysis. The algorithm may be implemented by using any software capable of solving MIP problems. To the best of our knowledge there are no other implementations of algorithms to solve the multiparametric min max 0-1-IP problem relative to the very general objective function considered and for this reason we did not compare the performance of our algorithm with any other.

We prefer to think that the multiparametric algorihtm is a general metodology and problems $Q^{(r)}$ would be solved with specialized algorithms associated to the 
structure of the non-parametric problems $P(\theta)$ in order to solve hard problems with higher dimensions and more parameters if necessary.

In a near future we will intend to develop a procedure to generate the nondominated set of a multiobjective 0-1-IP problem by using our multiparametric algorithm. In order to present some basic theory of multiobjective programming we use the presentation of Alves and Climaco [3] as follows:

Consider the following multiobjective 0-1-IP problem:

$$
(P) \quad \max c^{(1)^{t}} x, \cdots, c^{(q)^{t}} x \text { s.t. } x \in X .
$$

A solution $\bar{x}$ is efficient for problem $P$ if and only if there is no $x \in X$ such that $c^{(i)^{t}} x \geq c^{(i)^{t}} \bar{x}$ for all $i=1, \cdots, q$ and $c^{(i)^{t}} x>c^{(i)^{t}} \bar{x}$ for at least one $i$.

Let $X_{E}$ be the set of efficient solutions $\left(X_{E}=\{x \in X: x\right.$ is efficient $\left.\}\right)$.

A solution $\bar{x}$ is weakly efficient for problem $P$ if and only if there is no $x \in X$ such that $c^{(i)^{t}} x>c^{(i)^{t}} \bar{x}$ for all $i=1, \cdots, q$.

Let $X_{W}$ be the set of weakly efficient solutions $\left(X_{W}=\{x \in X: x\right.$ is weakly efficient $\}$ ).

Let $Z \subset \mathbb{R}^{q}$ be the image of the feasible set $X$ in the objective functions space (the criteria space). A point $z$ corresponding to a efficient (weakly efficient) solution $x$ is called non-dominated (weakly non-dominated).

Let $Z_{E}$ be the non-dominated set defined as follows:

$$
Z_{E}=\left\{z \in \Re^{q}: \text { there exists } x \in X_{E} \text { such that } z_{i}=c^{(i)^{t}} x(i=1, \cdots, q)\right\} \cdot
$$

Let $Z_{W}$ be the weakly non-dominated set defined as follows:

$$
Z_{W}=\left\{z \in \Re^{q}: \text { there exists } x \in X_{W} \text { such that } z_{i}=c^{(i)^{t}} x(i=1, \cdots, q)\right\}
$$

Let $\bar{C}$ denotes a reference point of the criteria space: $\bar{C}_{i}>c^{(i)^{t}} x$ for all $x \in X$ and for all $i=1, \cdots, q$.

The $\theta$-weighted Tchebycheff norm computed in $x \in X$ is defined as: $\max \left\{\theta_{i}\left(\bar{C}_{i}-\right.\right.$ $\left.\left.c^{(i)^{t}} x\right): i=1, \cdots, q\right\}$ where $\theta \in \Omega=\left\{\theta: \theta \in \mathbb{R}^{q}, \quad \theta \geq 0, \sum_{i=1}^{q} \theta_{i}=1\right\}$.

Bowman [5] proved that the multiparametric analysis of:

$$
(P(\theta)) \min \max \left\{\theta_{i}\left(\bar{C}_{i}-c^{(i)^{t}} x\right): i=1, \cdots, q\right\}
$$

generates the non-dominated set as follows: for all $\bar{z} \in Z_{E}$ there exists

$\bar{\theta} \in \Omega$ such that $\bar{z}_{i}=c^{(i)^{t}} \bar{x}(i=1, \cdots, q)$ where $\bar{x} \in X_{E}$ is an optinal solution of $P(\bar{\theta})$.

However, some weakly non-dominated solutions may appear if there exists $\bar{\theta} \in \Omega$ such that the optinal solution of $P(\bar{\theta})$ is not an efficient solution. 
The generation of weakly non-dominated solutions is avoided by considering the augmented $\theta$-weighted Tchebycheff norm (Steuer and Choo [24]) and the corresponding multiparametric analysis:

$$
\left(P(\theta) \min \max \left\{\theta_{i}\left(\bar{C}_{i}-c^{(i)^{t}} x\right)-\alpha \sum_{i=1}^{q} c^{(i)^{t}} x: i=1, \cdots, q\right\}\right.
$$

where $\alpha$ is small enough positive value.

As you can see our algorithm may be used to generate the non-dominated set. However some computational experience suggests that the linearization method must be carefully designed. Also, an approach to generate a representative subset of the non-dominated set may be designed like in other generating methods (Sylva and Crema [25], Sylva and Crema [26], Sylva and Crema [27], Laumanns et al. [18]).

Acknowledgements. The financial assistance by CDCH-UCV project (PI 03-00-6301-2006) made possible the research upon which this paper is based and is gratefully acknowledged. We also thank the referees for their helpful comments.

\section{REFERENCES}

[1] W.P. Adams and R.J. Forrester, A simple recipe for concise mixed 0-1 linearizations. Oper. Res. Lett. 33 (2005) 55-61.

[2] W.P. Adams, R.J. Forrester and F.W. Glover, Comparisons and enhancement strategies for linearizing mixed 0-1-quadratic programs. Discrete Optim. 1 (2004) 99-120.

[3] M.J. Alves and J. Climaco, A review of interactive methods for multiobjective integer and mixed-integer programming. Eur. J. Operat. Res. 180 (2007) 99-115.

[4] H. Arsham, http://home.ubalt.edu/ntsbarsh/Business-stat/Refop.htm.

[5] V.J. Bowman Jr, On the relationship of the Tchebycheff norm and the efficient frontier of multiple-criteria objectives. in Multiple Criteria Decision Making, edited by H. Thiriez, S. Zionts, Lect. Notes Economics Math. Systems, edited by H. Thiriez, S. Zionts, SpringerVerlag, Berlin (1976) 76-86.

[6] A. Crema, A contraction algorithm for the multiparametric integer linear programming problem. Eur. J. Oper. Res. 101 (1997) 130-139.

[7] A. Crema, An algorithm for the multiparametric 0-1-integer linear programming problem relative to the objective function. Eur. J. Oper. Res. 125 (2000) 18-24.

[8] A. Crema, An algorithm for the multiparametric 0-1-integer linear programming problem relative to the constraint matrix. Oper. Res. Lett. 27 (2000) 1-46.

[9] A. Crema, The multiparametric 0-1-Integer Linear Programming problem: A unified approach. Eur. J. Oper. Res. 139 (2002) 511-520.

[10] A.M. Geoffrion and R. Nauss, Parametric and postoptimality analysis in integer linear programming. Manag. Sci. 23 (1977) 453-466.

[11] H.J. Greenberg, An annoted bibliography for post-solution analysis in mixed integer propgramming and combinatorial optimization, Advances in Computational and Stochastic Optimization, Logic Programming and Heuristic Search, edited by David L. Woodruff, Kluwer Academic Publishers, Boston, MA (1998) 97-148.

[12] H.J. Greenberg, An annoted bibliography for post-solution analysis in mixed integer programming and combinatorial optimization, http://www-math.cudenver.edu/ ${ }^{\sim}$ greenbe/ papers/mipbib.pdf. 
[13] IBM Optimization Library C, Application Programming Interface. Available at http:// www-306.ibm.com/software/data/bi/osl/pubs/library/featCAPI.htm.

[14] L. Jenkins, Parametric mixed integer programming: an application to solid waste management. Manag. Sci. 28 (1982) 1270-1284.

[15] L. Jenkins, Using parametric integer programming to plan the mix of an air transport fleet. INFOR 25 (1987) 117-135.

[16] L. Jenkins, Parametric-objective integer programming using knapsack facets and Gomory cutting planes. Eur. J. Oper. Res. 31 (1987) 102-109.

[17] L. Jenkins, Parametric methods in integer linear programming. Ann. Oper. Res. 27 (1990) 77-96.

[18] M. Laumanns, L. Thiele and E. Zitzler, An efficient, adaptive parameter variation scheme for metaheuristics based on the epsilon constraint method. Eur. J. Oper. Res. 169 (2006) 932-942.

[19] Z. Li and M.G. Ierapetritou, A new methodology for the general multiparametric mixed integer linear programming (MILP) problems. Ind. Eng. Che. Res. 46 (2007) 5141-5151.

[20] S. Martello and P. Toth, The bottleneck generalized assignment problem. Eur. J. Oper. Res. 83 (1995) 621-638.

[21] Optimization Soubroutine Library, release 2, Guide and Reference, IBM (1992).

[22] M. Oral and O. Kettani, A linearization procedure for quadratic and cubic mixed integer problems. Oper. Res. 40 (1992) S109-S116.

[23] J.L. Quintero and A. Crema, An algorithm for multiparametric min max 0-1-integer programming problem relative to the objective function. RAIRO Oper. Res. 39 (2005) 243-252.

[24] R.E. Steuer and E.U. Choo, An interactive weighted Tchebycheff procedure for multiple objective programming. Math. Program. 26 (1983) 326-344.

[25] J. Sylva and A. Crema, A method for finding the set of non-dominated vectors for multiple objective integer linear programs. Eur. J. Oper. Res. 158 (2004) 46-55.

[26] J. Sylva and A. Crema, A method for finding well-dispersed subsets of non-dominated vectors for multiple objective mixed integer linear programs. Eur. J. Oper. Res. 180 (2007) 10111027.

[27] J. Sylva and A. Crema, Enumerating the set of non-dominated vectors in multiple objective integer linear programming. RAIRO Oper. Res. 42 (2008) 371-388.

[28] B. Thiongane, A. Nagih and G. Plateau, Theoretical and algorithmic study for parametric 0-1 linear programs relative to the objective function. Research Report LIPN 2003-01. 